\title{
Early neoplasia of the common bile duct diagnosed and completely removed using multiple endoscopic modalities
}
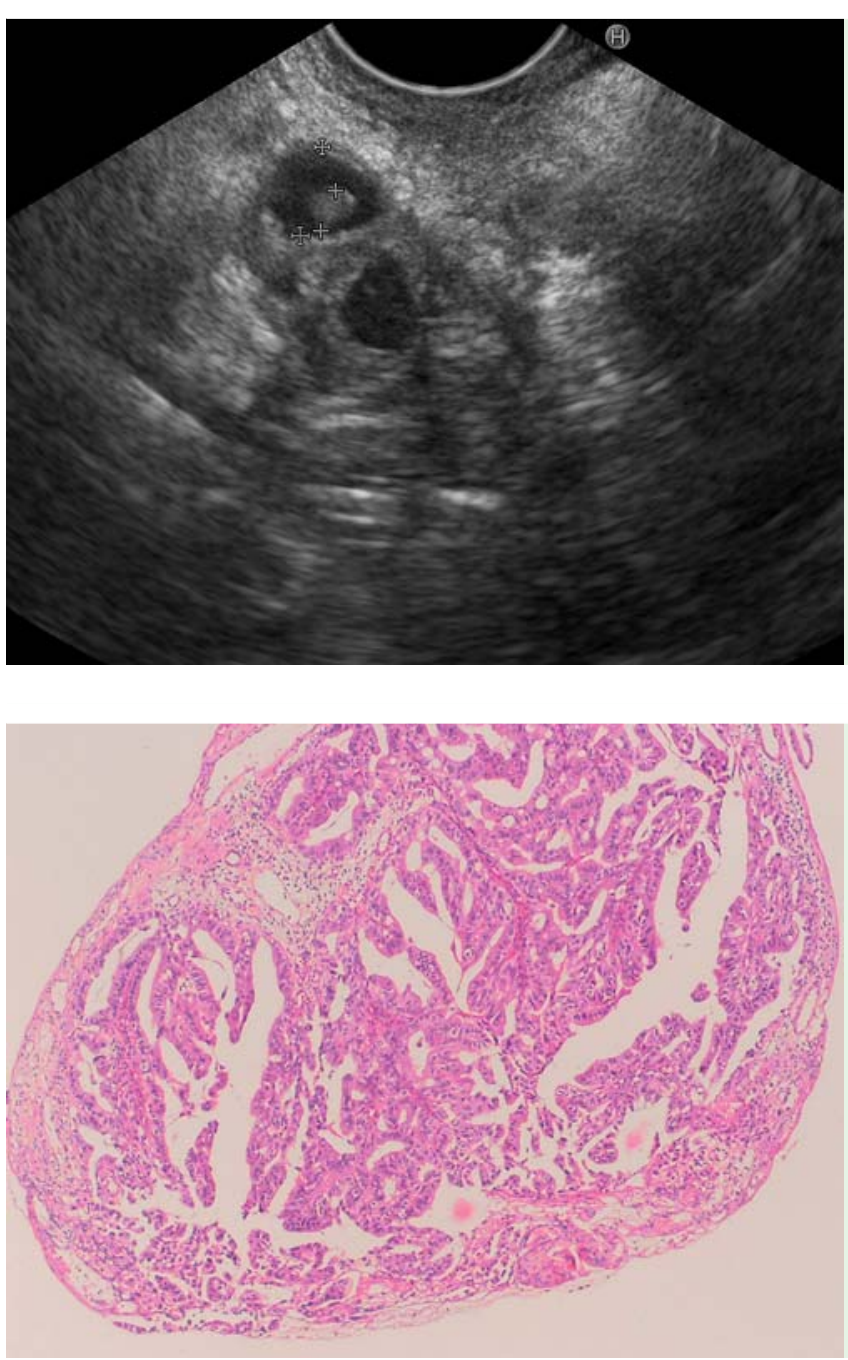
grade dysplasia and a

We report the case of a 73-year-old man who presented with a recurrent liver abscess, the second episode occurring after an interval a few months. Blood cultures were positive for Klebsiella pneumoniae on both admissions. He underwent magnetic resonance cholangiopancreatography (MRCP), which revealed a mildly dilated common bile duct (CBD) without any definite cause of obstruction. He was therefore scheduled to undergo further evaluation by endoscopic ultrasound (EUS), for which he was sedated in the endoscopic suite using intravenous propofol. Linear EUS (EG-3870UTK, Pentax, Miyaki, Japan) showed a 5-mm polypoid mass in the mid to distal portion of the CBD ( Fig.1). The gallbladder showed
Fig. 1 Endoscopic ultrasound (EUS) view showing a 5-mm polypoid mass in the mid to distal portion of the common bile duct.

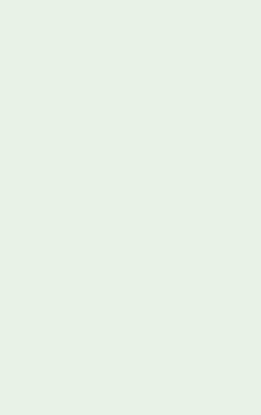

Fig. 3 Histopathology of the removed polypoid mass showing high glandular pattern without definite stromal invasion.

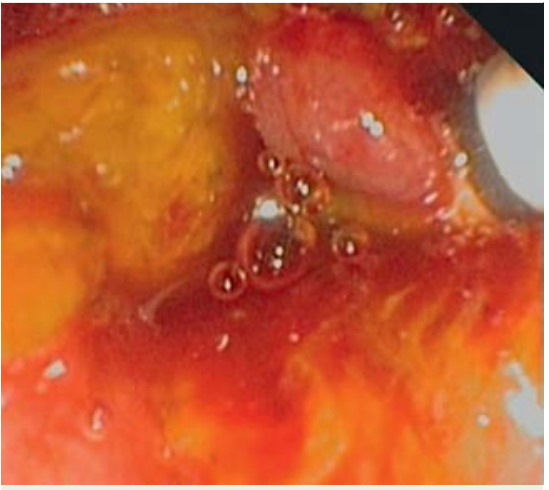

Fig. 2 Endoscopic view during endoscopic retrograde cholangiopancreatography (ERCP) showing the polypoid mass that popped out during balloon extraction.

Direct per oral cholangioscopy was performed using an ultraslim gastroscope (GIF-N180, Olympus), and the residual stalk of the polyp was identified in the mid to distal CBD ( Fig.4a,b). Narrow band imaging showed normal mucosa and a normal vascular pattern at the base of the lesion ( Fig.4c, d). Multiple biopsies were however obtained, histopathology of which showed normal bile duct epithelium without evidence of residual neoplasm.

CBD adenomas are rare benign neoplasms of the bile duct [1-3], although there has been a report of a bile duct adenoma giving rise to an adenocarcinoma [4]. To date, there have been only a few case reports on the endoscopic treatment of bile duct adenomas. In our patient, the lesion was a premalignant CBD adenoma that was successfully treated using multiple endoscopic modalities.

Endoscopy_UCTN_Code_TTT_1AR_2AF

\section{Competing interests: None}

\section{N. Sirimontaporn' ${ }^{1}$ P. Aswakul ${ }^{1}$, P. Junyangdikul ${ }^{2}$, V. Prachayakul ${ }^{3}$} (ه Fig. 2). This lesion was removed using biopsy forceps and histopathology showed it to be an adenoma with high grade dysplasia and an ill-defined margin ( Fig.3a). As a result, the patient was scheduled for a cholangioscopy to further evaluate the base of the adenoma.
1 Liver and Digestive Institute, Samitivej Sukhumvit Hospital, Bangkok, Thailand

2 Department of Pathology, Samitivej Srinakarin Hospital, Bangkok, Thailand ${ }^{3}$ Siriraj GI Endoscopy Center, Siriraj Hospital, Bangkok, Thailand 

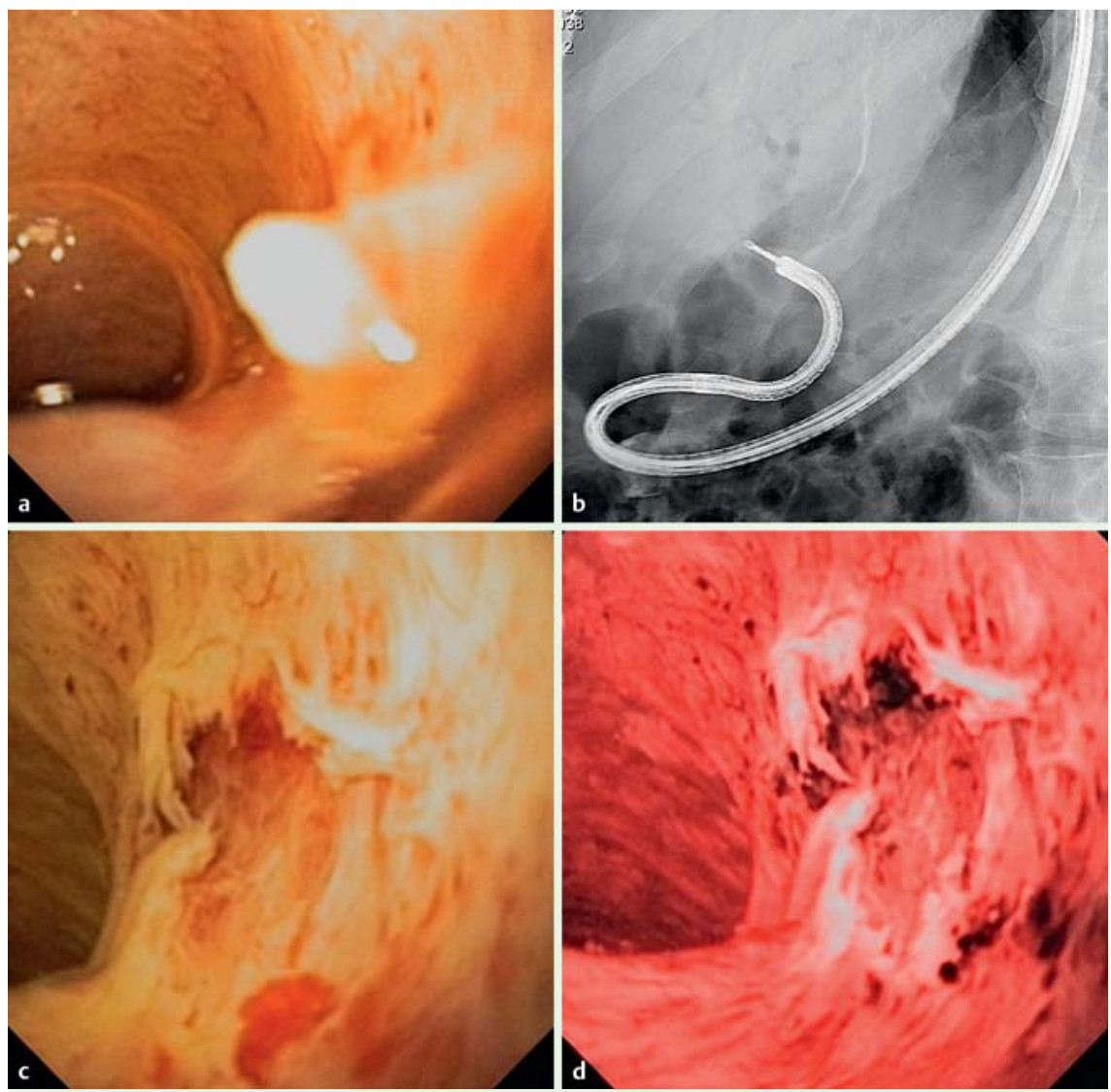

Fig.4 Images obtained during cholangioscopy to further evaluate the stalk of the polyp in the common bile duct showing: a the initial cholangioscopic view; $\mathbf{b}$ a fluoroscopic image of the ultraslim gastroscope in the common bile duct $\mathbf{c}$ a cholangioscopic view of the biopsy site; $\mathbf{d}$ a cholangioscopic view of the biopsy site with narrow band imaging, which revealed normal mucosa and vascular pattern.

\section{References}

1 Yang J, Wang $W$, Yan $L$. The clinicopathological features of intraductal papillary neoplasms of the bile duct in a Chinese population. Dig Liv Dis 2012; 44: $251-256$

2 Koga F, Tanaka H, Takamutsu S et al. A case of very large intrahepatic bile duct adenoma followed for 7 years. World J Clin Oncol 2012; 3: $63-66$

3 Saleem A, Wang KK, Baron TH. Successful endoscopic treatment of intraductal extension of a villous adenoma with high-grade dysplasia, with 3-years follow-up. Gastrointest Endosc 2011; 74: 714-716

4 Munshi AG, Hassan M. Common bile duct adenoma: case report and brief review of literature. Surg Laparosc Endosc Percutan Tech 2010; 20: e193 - e194

\section{Bibliography}

DOI http://dx.doi.org/

$10.1055 / \mathrm{s}-0032-1326278$

Endoscopy 2013; 45: E102-E103

(C) Georg Thieme Verlag KG

Stuttgart · New York

ISSN 0013-726X

\section{Corresponding author \\ V. Prachayakul, MD}

Division of Gastroenterology,

Department of Internal Medicine

Siriraj Hospital

Mahidol University

Bangkok 10700

Thailand

Fax: +66-2419-6101

kaiyj@@gmail.com 